\title{
A Review on Analysis of Effect of Yoga and Meditation using Current Technology
}

\author{
Shrivatsa D. Perur \\ Assistant Professor \\ Dept. of ISE, \\ GIT, Belagavi
}

\author{
Harish H. Kenchannavar, $\mathrm{PhD}$ \\ Professor \\ Dept. of ISE, \\ GIT, Belagavi
}

\begin{abstract}
Nowadays people are more oriented towards their health and fitness and yoga has been serving their purpose in a positive way. Yoga is a type of exercise in which you move your body into various positions in order to become more fit or flexible, to improve your breathing and to relax your mind. The technology has also evolved to the extent that people find every solution for their issues using the technology. It makes the life of people easier. This paper gives an overview of how yoga and technology can be brought together so that it helps the folks to choose the appropriate techniques of yoga to overcome their health issues
\end{abstract}

\section{General Terms}

Yoga and Technology, Interactive app for yoga

\section{Keywords}

Cognitive neuroscience, Meditation analysis, EEG analysis

\section{INTRODUCTION}

Yoga is an ancient science derived from India. Practicing Yoga could make our brain keeping activity and physical function reaching to harmonious unity, thus promoting our physical health and improving the feeling of happiness[1][2]. Therefore, Yoga is being recognized more and more important as an old science.

The World Health Organization defined health in its broader sense in 1946 as "a state of complete physical,mental, and social well-being and not merely the absence of disease or infirmity." Seen from this definition, health depends on physical, mental and social well-being. Today, Yoga has become ancient health culture. As a relieving and relaxing exercise, Yoga has become one of the most fashionable body building exercises and is very popular with youngsters. As a static exercise, Yoga regulates bodies into good condition through posture, breathing, meditation and so on, and plays an important role in our physical, mental, spiritual and emotional health. In many countries, Yoga is taken as a method for treating diseases, such as asthma, diabetes, hypertension, arthritis, indigestion and so on.

Yoga is very popular because it may balance human beings' mental system and endocrine system, thus exerting direct influence on human beings' other systems and getting them balanced. For modern city women and men, Yoga may regulate their emotions, relieve them of their stress resulting from fast life pace, eliminate the toxin in bodies, increase their energy, and burn the fat to keep fit, get into shape and preserve our health. Yoga is applicable tohuman beings of all ages, and helps to reduce the load on heart. More and more human beings expect to have their health improved, their bad emotions and ill temper eliminated and their mental, physical and social adaptability improved through Yoga. Men may be mentally and physically regulated by practicing Yoga to be adapted to the society better and have their work and quality of life improved. Yoga is the oldest way for strengthening bodies and the most fashionable way for body building, which focuses on "combining healthy spirit with healthy bodies" so that we may be physically and mentally harmonious.

In addition, Yoga helps us to pick up the quality of keeping calm and objective, the attitude of taking things as they are, sturdiness and gracefulness so that you may get the joy and happiness of life. Yoga is to reach proper positions in a comfortable way stably by moving slowly, flexibly at ease to be stretched to the maximum extent by consuming the least energy. Yoga consists of a lot of postures, about 20-30 in practice. For Yoga, the harmony depends on your concentration on your practice instead of your nice posture. Everyone may practice Yoga, and may get into a good condition through different postures. Yoga is characterized in that those who practice Yoga may enjoy the process both physically and mentally, and is good for bodies, minds, spirit, emotions and so on.

According to medical explanation, Yoga is good because it may balance nervous system and endocrine system of human bodies, thus exerting influence on other systems and reaching a balance as a whole beauty in appearance instead of the innermost being. By contrast, while Yoga shapes you, it may also provide you with internal power. After a period of Yoga exercise, you will be surprised to find that your innermost being has been changed. You will not torture yourself for the purpose of losing weight and you will be beautiful because you are happy, thus being physically and mentally trained without any auxiliary body building instruments or tools.

Neither yoga is like other body building sports that are limited by the field such as ball, swimming and so on. You shall not just spend some time on practicing asana or meditation, instead, the concept of Yoga shall be reflected throughout your life. For instance, you shall select natural environment for living, bland diet, relaxing music, become outgoing and calm.

Yoga is never like general body building sports, and advocates the life philosophy of "harmony of body and mind". 


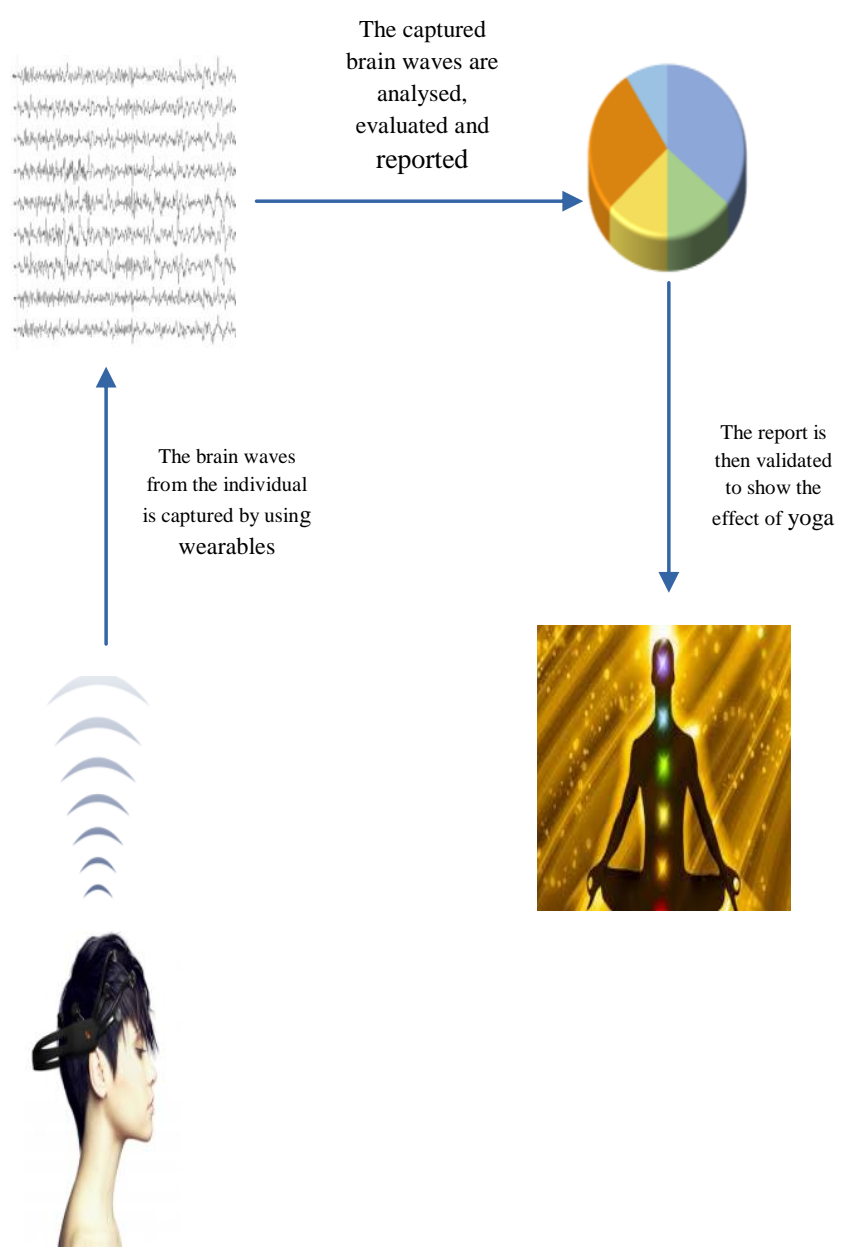

Fig 1: Analyzing effect of yoga on an individual

Besides bringing physical harmony and health in all aspects to human beings, Yoga also helps human beings to be dedicated, calm, objective, cultivate our morality, and to become both physically and mentally healthy, which is the greatest advantage of Yoga that other sports do not have.

\section{PROBLEM STATEMENT}

The effectiveness of yoga on an individual's physical and mental health is to be evaluated in the work. The work deals with analysis of the effect of yoga on individual can increase the health of person. Concentration is made on analysing what causes the changes in the health of a person undergoing yoga therapy on a longer duration. The use of few data mining algorithms to validate and evaluate the effect is to be considered

\section{LITERATURE SURVEY}

Zhaoyuan [1] opines that yoga is a healthful aerobic exercise exclusive to women and men. Yoga relieves pressure to a considerable level in the modern fast paced work and life. Authour performs a research into Yoga's functions for men's health and its influence on men's bodies and minds, and through the research, it is found that Yoga may effectively activate body fat, regulate the heart rate, reduce the load on heart and vitalize men in regard to their physical health so that they may work and live a life confidentially; Yoga also may help to improve their interpersonal relationship in regard to improving men's social adaptability so that they may work healthily, deal with others properly, and eliminate bad mental factors, thus improving individuals' charm.
$\mathrm{Wu}$ et. $\mathrm{Al}[2]$ opines that Yoga exercise could promote our physical health and improve the feeling of happiness, so it is becoming more and more popular and the establishment of Yoga learning and self-instruction expert system has great significance for the popularization and spread of Yoga. In the research, the author analyses the Yoga training knowledge is analyzed using POAD expert system model, Yoga learning and self-instruction expert system is established taking Yoga training technique problem as framework, the definitions of MP4 objects in Yoga knowledge are given, system operation mechanism is designed accustomed to Yoga training, and Yoga learning and self-instruction expert system Yoga Expert is developed.

Baglio et. $\mathrm{Al}[3]$ in their work studied the spatial power distribution in Magnetoencepbnlography (MEG) data collected in a subject performing two different yoga breathing exercises specific for the treatment of obsessive compulsive disorder. They reconstructed, in relation with all the frequency components, a characteristic spatial structure of power distribution using the correlation function for both the recordings. through the power spectral analysis, the characterization of the gamma-band activity was carried out on allowing to underline the differences due to the activities of different phases of the same protocol and the effects of performing different breathings exercises.

Howorka et. $\mathrm{Al}[4]$ in their work suggested that Experience with frequency domain analysis of the heart rate variability represents an effective non-invasive tool for cardiac risk stratification and assessment of autonomic function e.g. in diabetes and hypertension, in both the experimental and clinical settings. The method proposed could be used to assess the acute influence of yoga and short-time moderate exercise on the autonomic cardiac regulation. They demonstrated the immediate decrease of sympathetic and increase of parasympathetic activity after yoga.

Yunhai et. $\mathrm{Al}[5]$ studied the complexity of heartbeat dynamics during specific traditional forms of Chinese Chi and Kundalini Yoga meditation in healthy young adults. The technique based on the algorithm of permutation entropy to heartbeat interval time series was analysed. The entropy of these oscillations during meditation was significantly less than those in the pre-meditation state. The results show'it is feasible that permutation entropy (PE) index can be used to analyze the effect of Chi and Yoga to restrain HRV

Jae Won Bang et. $\mathrm{Al}[6]$ proposed a new method that combines an EEG acquisition device and a frontal viewing camera to isolate and exclude the sections of EEG data containing these noises. This method is novel in the following three ways. First, the accuracy of detecting head movements based on the features of EEG signals in the frequency and time domains and on the motion features of images captured by the frontal viewing camera is compared. Second, the features of EEG signals in the frequency domain and the motion features captured by the frontal viewing camera are selected as optimal ones. The dimension reduction of the features and feature selection are performed using linear discriminant analysis. Third, the combined features are used as inputs to support vector machine (SVM), which improves the accuracy in detecting head movements. The experimental results of authors show that the proposed method can detect head movements with an average error rate of approximately $3.22 \%$, which is smaller than that of other methods.

Vijayaragavan et. Al[7] proposed the idea to develop a passage that serves as an effective solution to overcome this 
problem by providing people with a smart phone application based integrated solution which makes use of Yoga and Music Therapy techniques. The objective was to bring about relaxed state of mind by monitoring the EEG readings of a person while subjecting him to Yoga \& Music therapy simultaneously through an efficient Android Application which can restore the calm state of mind for the user during any stressed conditions.

Shaw et. Al[8] In their work did a comparative study to classify the resting brain state associated with Kriya Yoga meditation practice using SVM and Kernel-SVM (k-SVM). The EEG signals were captured from ten non-meditators (control group) and 23 meditators group. The results of both SVM and k-SVM were compared in both the groups. Additionally, the average classification accuracy was found to be $85.543 \%$ for SVM and $90.8259 \%$ for k-SVM. The obtained results showed that the kernel-based SVM surpassed the conventional SVM in classifying the meditation and nonmeditation allied EEG.

Godse et. al[9] The aim of the present research was to study the effects of suryanamaskar on R-dispositions among college students with high stress. Participants eligible for the study, that is, high on stress symptoms were randomly assigned to suryanamaskar and control group. Differences on various Rdispositions and stress dispositions were studied. The mean differences for the subscales indicate that sleepiness and stress reduced as a disposition and there was an increase in physical relaxation, mental quietness, ease and peace, being refreshed, strength and awareness, and joy. The observed effects on the above stated R-dispositions can be attributed to components of suryanamaskar. It is clear that a 2 weeks program helps participants to reach the state of a quiet mind at a dispositional level.

Woodyard [10] in thier work says that yoga is rapidly emerging in the Western world as a discipline for integrating the mind and body into union and harmony. when adopted as a way of life, yoga improves physical, mental, intellectual and spiritual health. Yoga offers an effective method of managing and reducing stress, anxiety and depression and numerous studies demonstrate the efficacy of yoga on mood related disorders.

Jagannathan et. $\mathrm{Al}[11]$ describes the development of a yoga program aimed to reduce burden and improve coping of family caregivers of inpatients with schizophrenia in India. This study was one of the first studies to use a sound methodology of inductive enquiry model for the development of a need-based yoga program for caregivers of in-patients with schizophrenia in India. The findings were highly indicative and future studies could test the efficacy of the program with a larger quantitative sample to reconfirm its validity.

Ramanathan et. $\mathrm{Al}[12]$ undertook a study to evaluate the effectiveness of yoga on the mental health status of elderly women inmates residing in a hospice in Pondicherry. The influence of yoga in the reduction of depression and anxiety scores and improvement in self-esteem scores in elderly women subjects was evident from this study. This may be attributed to changes in central neurotransmitters such as gamma-amino butyric-acid coupled with increased parasympathetic tone and decreased sympathy-adrenal activity. It was recommended that yoga should be a part of health-care facilities for elderly as it could enhance the quality of life by improving their overall mental health status. It could provide a healthy and positive alternative from depressing negative thoughts, and give them a sense of purpose and hope, reliability, and generalizability.

Moliver et. Al[13] examined the extent to which psychological attitudes, transcendence, mental mastery, and subjective vitality in a sample of female yoga practitioners over 45 years varied according to the length and frequency of yoga practice. There were significant positive relationships between yoga experience and all outcome variables. These significant relationships remained after accounting for age and lifestyle factors. When authors computed yoga experience in terms of total calendar years, without accounting for hours of practice, significant relationships did not remain. Transcendence of the ordinary was the most strongly associated with current yoga practice frequency, and positive psychological attitudes were the most strongly associated with total lifetime hours of practice.

Hawkins et. $\mathrm{Al}[14]$ investigated the impact of participating in yoga classes on the amount of exercise behaviour and perception of physical exertion when compared to nonstructured exercise sessions between two young adults with ID in a post-secondary education setting. The data from this study indicated a functional relationship between participation in yoga exercise classes and amount of exercise behaviour. There was a sharp increase in physical exertion for both subjects when they participated in a yoga exercise program as opposed to non-structured exercise. The author opined that if there was increase in exercise and continued over an extended period of time,it might help in reducing the likelihood of developing chronic health diseases and improving physical fitness levels. [15] Enjoyment during the yoga intervention remained within an enjoyable range and both students voluntarily continued attending yoga classes upon the conclusion of the study.

Researchers at Teesside University in the U.K. investigated how combining cognitive activities and elements of yoga, tai chi, qigong and meditation with routine physical exercise affected dementia patients. They found that a holistic exercise program focusing on both mind and body can help improve quality of life for dementia patients.[16]

Ansari [17] in the work opined that Kapalabhati effectively targets and combats the features of MS. Since PCOS and MS share a common pathogenic pathway, it was worthwhile to consider that Kapalabhati can be effective against the features of PCOS too, though there was a dearth of studies and literature in this regard. On a positive note, Kapalabhati would help to address infertility in PCOS women, a main cause of concern in women of reproductive age.

Khemka et. Al[18] studied on evaluation of the effects of integral yoga practices on these variables and also the consistency of correlations observed between them. Significant pre-post changes were found in all variables. Significant correlations were found between the following pairs: The two sustained attention variables; emotional intelligence and general health; GHQ and tamas; sattva and tamas; and rajas and tamas. The study showed that there were significant changes in all variables $(\mathrm{P}<0.001)$ except in sattva. It also confirmed that EQ and general health variables correlate significantly with each other and negatively with tamas. EQ and tamas form positive and negative predictors of health respectively. Sattva correlates positively with EQ suggesting that a sattvic personality indicates better selfcontrol. This suggests that, by improving guna personality, long-term yoga practice may stabilize EQ. 
Gururaja et. $\mathrm{Al}[19]$ conducted a study in Japan to find the effect of yoga on mental health between young and senior people. Reduction in State and Trait anxiety score signifies that yoga has both immediate as well as long-term effect on anxiety reduction. Thus author opines that yoga helps to improve the mental health in both the groups.

Jimenez et. $\mathrm{Al}[20]$ suggested that $\mathrm{HY}$ asanas, practiced as a systematic physical activity andconducted by an expert instructor in untrained and aging individuals, can improve health and serve as the basis for a physically active lifestyle.

Deshpande et. $\mathrm{Al}[21]$ demonstrated that an eight week intervention of an integrated yoga module decreased verbal aggressiveness in the yoga group (in males and those below 25 years of age), with a no significant increase in the PE group.

\section{METHODOLOGY}

Stage1: Sample collection from faculty of Engineering college

At the initial stage, Participants/ Subjects are identified from in and around the institution. Using the device EMOTIV $\mathrm{EPOC}+($ a 14 channel portable and handy brain activity map capturing instrument)/ Brainsense/Neurosky mindwave mobile, the EEG signals and brain activity map is captured before the subject is performing the pranayama. Along with this, a response for a self administered questionnaire is also collected from subjects. The faculty are asked to wear the devices for a period of six hours a day for the data or sample collection.

\section{Stage 2: Analysis of Data}

The collected brain activity map and EEG signals are analyzed by converting the analog data into digital format. The data obtained here will be a single valued data with the time stamp. The EEG data is analyzed with the help of a neurologist and cognitive behavior of a person is studied with the help of a psychologist. The digitalized EEG data is assessed and analyzed using a method called one way ANOVA(Analysis of Variance). Along with this, the responses an individual gives for the self administered questionnaire is also analyzed by Spielberger's State-Trait Anger Expression. The data analyzed is kept as training set and the subject is made to undergo yoga therapy or meditation therapy for a continuous period. During this again the procedure is repeated so as to analyze the changes happening in the individual.

\section{Stage 3: Finding the inferences}

The EEG signals and brain activity map is again collected from the subjects while performing yoga/pranayama which serves us as the test data. After the stipulated time again, the individual is tested for the EEG regularly to analyze the changes in the health of a person. The result from EEG and questionnaire is analyzed by combining them and the transformation in reducing anger, anxiety and tension that has been observed in an individual is exhibited.

\section{Stage 4: Development of intelligent system}

After the analysis of the data or samples obtained, a prototype is modeled so as to help an individual to control his emotions. An IoT device would be prepared which detects the state of emotions of a person and at a preliminary stage would give an alarm to the individual to control his state of emotion.

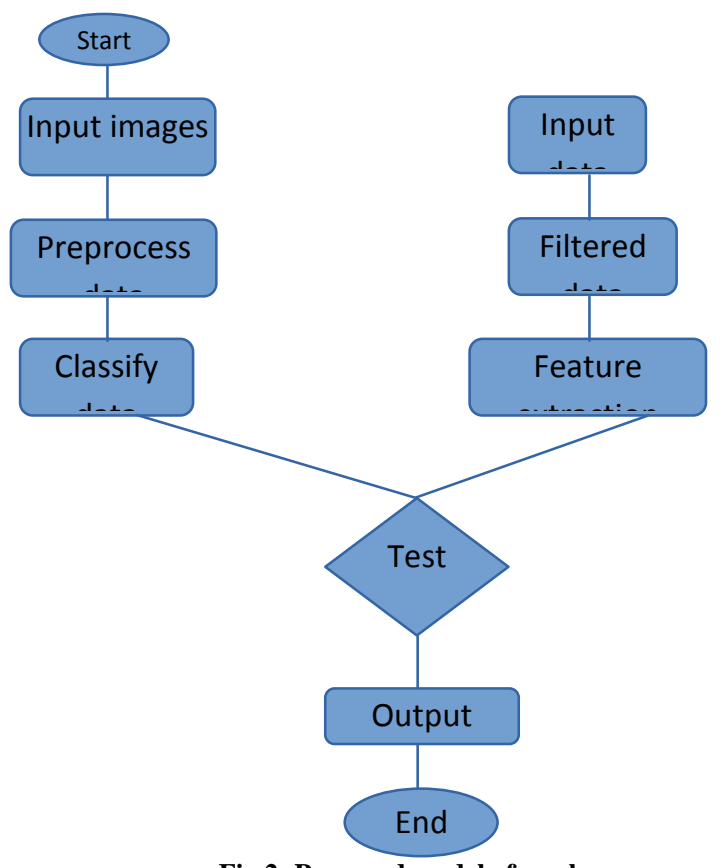

Fig 2: Proposed model of work 


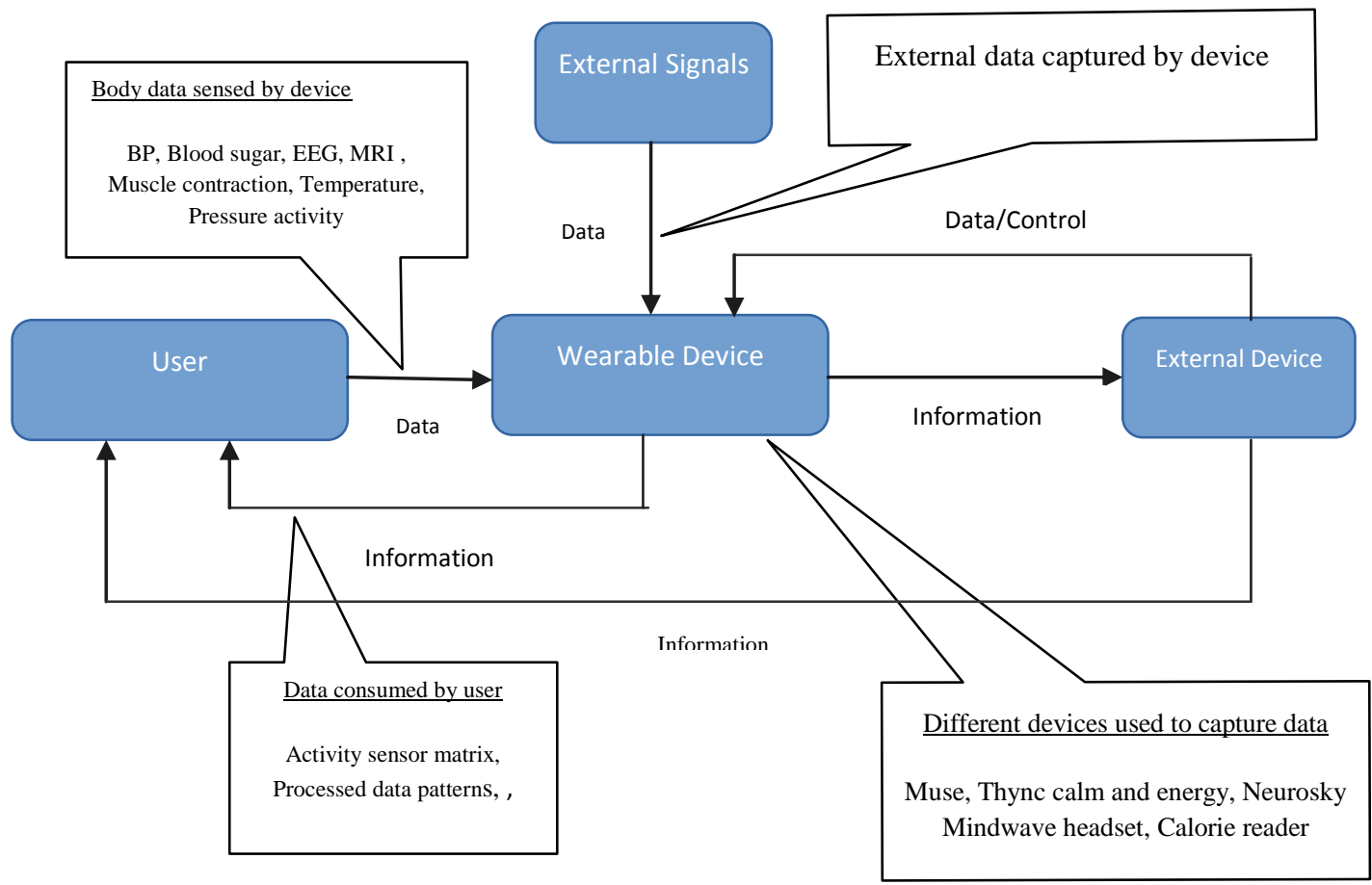

Fig 1: Working model

\section{CONCLUSION}

People are looking out in positive way of living by keeping their health in a good condition. To improve breathing and relax, people tend to use the yogic way of exercising which provides a way to overcome every health issue in a natural way by strengthening the immune system. The technology has improved drastically and every single thing can be found using means of technology. In this paper an overview of how yoga and technology can be brought together is given, an idea to produce a interactive system so as to help people control their emotions using yoga and get the best out of them.

\section{ACKNOWLEDGMENTS}

Our sincere thanks to the Principal of the institute who has been our support for the work being carried out, experts who have contributed towards the work.

\section{REFERENCES}

[1] Huang Zhaoyuan, "Research into Yoga's Promoting Functions for Men's Health", International Conference on Human Health and Biomedical Engineering, Jilin, China, August 2011, pp 19-22

[2] Wei Wu, Wensheng Yin, Feifei Guo, "Learning and Self-instruction Expert System For Yoga", IEEE, 2010

[3] S. Bagliol, M. Bucolo, L.Fortunal, M. Frascal, M. La Rosa, D. Shannahoff-Khalsa, "MEG Signals Spatial Power Distribution And Gamma Band Activity In Yoga Breathing Exercises", Proceedings of the Second Joint EMBSIBMES Conference Houston, TX, USA * October 2002, pp 175-176

[4] Howorka K. , Pumprla J., Heger G., Thoma H., Opavsky J., Salinger J., "Computerised Assessment of Autonomic Influences of Yoga Using Spectral Analysis of Heart Rate Variability", Proceedings RC IEEE-EMBS 8 14th BMESI 1995, pp 1.61-1.62

[5] Shen Yunhai, Bian Chunhua, Ma Qianli, "The Complexity of Heartbeat Dynamics During CHI and
YOGA Meditation", IEEE, 2011, pp 138-141

[6] Jae Won Bang, Jong-Suk Choi and Kang Ryoung Park, "Noise Reduction in Brainwaves by Using Both EEG Signals and Frontal Viewing Camera Images", Sensors, 2013, pp 6272-6294

[7] Gautham Raj Vijayaragavan, R.L.Raghav, K.P.Phani, Vivek Vaidyanathan, "EEG Monitored Mind Destressing Smart Phone Application using Yoga and Music Therapy", International Conference on Green Computing and Internet of Things (ICGCIoT), 2015, pp 412-415

[8] Laxmi Shaw, Aurobinda Routray, "A Critical Comparison Between SVM and k-SVM in the Classification of Kriya Yoga Meditation State-allied EEG”, IEEE International WIE Conference on Electrical and Computer Engineering (WIECON-ECE), Pune, India, December 2016, pp 19-21

[9] Anand Sharad Godse, Bhaskar R Shejwal, Amruta Anand Godse, "Effects of suryanamaskar on relaxation among college students with high stress in Pune, India", International journal for yoga, 2015, Volume 8 ,Issue 1, pp 15-21

[10] Catherine Woodyard, "Exploring the therapeutic effects of yoga and its ability to increase quality of life", International journla for yoga, 2011, Volume 4, Issue 2, pp 49-54

[11] Aarti Jagannathan, Ameer Hamza, Jagadisha Thirthalli, HR Nagendra, R Nagarathna, BN Gangadhar, "Development and feasibility of need-based yoga program for family caregivers of in-patients with schizophrenia in India, International journal for yoga, 2012, Volume 5, Issue 1, pp 42-47

[12] Meena Ramanathan, Ananda Balayogi Bhavanani, Madanmohan Trakroo, "Effect of a 12-week yoga therapy program on mental health status in elderly 
women inmates of a hospics", International journal for yoga, 2017, Volume 10, Issue 1, pp 24-28

[13] N Moliver, EM Mika, MS Chartrand, RE Haussmann, SBS Khalsa, "Yoga experience as a predictor of psychological wellness in women over 45 years", International journal for yoga, 2013, Volume 6, Issue 1, pp 11-19

[14] Brent L Hawkins, Joanna B Stegall, Madalyn F Weber, Joseph B Ryan, "The influence of a yoga exercise program for young adults with intellectual disabilities", International journal for yoga, 2012, Volume 5, Issue 2, pp 151-156

[15] "Overweight and obesity," Centers for Disease Control and Prevention. Available from: http://www.cdc.gov/obesity/defining.html. [Last accessed on 2010 June 21].

[16] " Study Shows Dementia Patients Benefit From Holistic Exercise Program”, Elsevier, 2014

[17] Reshma Mohamed Ansari, "Kapalabhati pranayama: An answer to modern day polycystic ovarian syndrome and coexisting metabolic syndrome?", International journal for yoga, 2016, Volume 9, Issue 2, pp 163-167

[18] Sushil S Khemka, Nagendra Hongasandra Ramarao, Alex Hankey, "Effect of integral yoga on psychological and health variables and their correlations", International journal for yoga, 2011, Volume 4, Issue 2, pp 93-99

[19] Derebail Gururaja, Kaori Harano, Ikenaga Toyotake, Haruo Kobayashi, "Effect of yoga on mental health: Comparative study between young and senior subjects in Japan”, International journal for yoga, 2011, Volume 4, Issue 1 , pp 7-11

[20] Arnulfo Ramos-Jimenez, Rosa P Hernandez-Torres, Abraham Wall-Medrano, Maria DJ Munoz-Daw, Patricia V Torres-Duran, Marco A Juarez-Oropeza, “ Cardiovascular and metabolic effects of intensive Hatha Yoga training in middle-aged and older women from northern Mexico", International journal for yoga, 2009, Volume 2, Issue 2, pp 49-54
[21] Sudheer Deshpande, HR Nagendra, Nagarathna Raghuram, “ Sudheer Deshpande, HR Nagendra, Nagarathna Raghuram", International journal for yoga, 2008, Volume 1, Issue 2, pp 76-82

[22] Bekiari A, Digelidis N, Hatzigeordiadis A, Sakelariu K. Development of a scale to assess verbal aggressiveness in the physical education context. Ital J Sport Sci 2005;12:160-4.

[23] Brown RP, Gerbarg PL. Sudarshan Kriya Yogic breathing in the treatment of stress, anxiety, and depression: Part I-neurophysiologic model. J Altern Complement Med 2005;11:189-201

[24] Harvey JR. The effect of yogic breathing exercises on mood. J Am Soc Psychosomat Dentist Med 1983;30:3948.

[25] Nagarathna R, Nagendra HR. Integrated approach of yoga therapy for positive health. 5th ed. Bangalore: SVYP; 2003.

[26] Kumari S, Nath NC, Nagendra HR. Enhancing emotional competence among managers through SMET, Psychological Studies. Natl Acad Psychol India 2007;52:171-3.

[27] Telles S, Naveen KV, Manoj D. Yoga reduces symptoms of distress in tsunami survivors in the Andaman islands. Evid Based Complement Alternat Med 2007;4:503-9.

[28] Salovey P, Mayor J, Brackett MA. Emotional Intelligence: Key readings on the Mayor and Salovey model. New York: Natl professional Resources Inc; 1995.

[29] Brown RP, Patricial LG. Sudarshan Kriya Yogic breathing in the treatment of stress, anxiety, and depression. Part II-clinical applications and guidelines. J Altern Complement Med 2005;11:711-7.

[30] Rani JN. Impact of yoga training on triguna and selfideal disparity. Psychological Studies. Natl Acad Psychol India 2007;52:174-7. 